\title{
Epidemiología de Lesiones Relacionadas con Colisiones de Vehículos Motorizados en dos Centros de Referencia del Suroccidente Colombiano. Reporte del Registro Internacional de Trauma de la Sociedad P
}

\author{
1 Juan Sebastian Calle-Toro, ${ }^{2}$ Carlos Ordonez, ${ }^{3}$ Álvaro I Sánchez, ${ }^{4}$ Juan Sanjuan, ${ }^{5}$ Marisol Badiel \\ ${ }^{6}$ Luis Pino, ${ }^{7}$ Rao R Ivatury, ${ }^{8}$ Michael Aboutanos
}

\begin{abstract}
RESUMEN
Introducción: Dado que las lesiones relacionadas con colisiones de vehículos motorizados son una causa de morbimortalidad prevenible y evitable, generan costos económicos y afectan calidad de vida, se justifica evaluar esta problemática en términos de resultados clínicos. El objetivo de este estudio es caracterizar la población de Cali con lesiones relacionadas con colisiones de vehículos motorizados que fueron atendidos en los servicios de urgencias de dos centros de alta complejidad de la ciudad.
\end{abstract}

Materiales y métodos: Estudio observacional descriptivo, utilizando un registro de trauma en dos hospitales de alta complejidad de Cali, Colombia.

Resultados: En un periodo de 8 meses 11889 atenciones secundarias a lesiones de causas externas intencionales y no intencionales fueron registradas. Del total de pacientes, 1395 $(11,6 \%)$ tenían lesión de tránsito, y de estos el $86 \%(n=1200)$ ingresaron con escala de severidad de trauma (ISS) $<9$. El $48,4 \%(n=675)$ de las atenciones estuvieron relacionadas

${ }^{1}$ Medical Student, ${ }^{2,8}$ Surgeon, ${ }^{3}$ Resident, ${ }^{4}$ Doctor, ${ }^{5}$ Doctor and Vice Chairman, ${ }^{6}$ Surgeon and Director, ${ }^{7}$ Professor (Emeritus)

${ }^{1}$ Department of Medicine, Universidad ICESI, Fundación Valle del Lili, Cali, Colombia

${ }^{2}$ Chief of Trauma and Acute Care Surgery Department, Hospital Universitario del Valle, Fundación Valle del Lili, Cali Colombia

${ }^{3}$ Department of General Surgery, Universidad CES, Fundación Valle del Lili, Cali, Colombia

${ }^{4}$ Department of Acute Care, Fundación Valle del Lili, Cali Colombia

${ }^{5}$ Department of Research, Clínica Universitaria Uribe, Uribe Cali, Colombia

${ }^{6}$ Department of Emergency, Hospital Universitario del Valle Cali, Colombia

${ }^{7}$ Division of Trauma, Critical Care and Emergency Surgery Commonwealth University Medical Center, Virginia, USA

${ }^{8}$ Chair, Division of Trauma, Critical Care and Emergency Surgery, Virginia Commonwealth University, Virginia, USA

Corresponding Author: Carlos Ordonez, Surgeon, Department of Acute Care Surgery, Hospital Universitario del Valle, Fundación Valle del Lili, Cali, Colombia, e-mail: ordonezcarlosa@gmail.com con el uso de motocicletas, el $26,8 \%(n=374)$, con uso de bicicletas y $10,3 \%(n=144)$ con vehículos livianos. El mecanismo de trauma contuso fue el más observado $(93,5 \%$, $n=1304)$. Estratificando los hallazgos por grupos de edad, que la motocicleta es el vehículo más relacionado, excepto en el grupo de edad de 5-14 años. Del total de pacientes, 777 $(55,7 \%)$ fueron hospitalizados y de ellos $114(14,7 \%)$ requirieron unidad de cuidado intensivo. La mortalidad global fue de 2,7\% (53 pacientes).

Conclusiones: En Cali, las personas que sufren más frecuentemente lesiones relacionadas con colisiones de vehículos motorizados son hombres jóvenes, económicamente activos, que usan motocicleta como medio de transporte, con sospecha de ingesta alcohol. Esperamos contribuir con próximas medidas y estudios.

Palabras claves: Vehicular, Motocicleta, Alcohol, Choque de vehÃculos de motor

How to cite this article: Calle-Toro JS, Ordonez C, Sánchez Ál, Sanjuan J, Badiel M, Pino L, Ivatury RR, Aboutanos M. Epidemiología de Lesiones Relacionadas con Colisiones de Vehículos Motorizados en dos Centros de Referencia del Suroccidente Colombiano. Reporte del Registro Internacional de Trauma de la Sociedad P. Panam J Trauma Crit Care Emerg Surg 2014;3(1):16-22.

Source of support: Nil

Conflict of interest: None

\section{ABSTRACT}

Background: Injuries related to motor vehicle crashes (MVCs) are an important source of preventable morbidity and mortality, generating significant social and economic burdens and increased costs to the health system. The objective of this study was to describe demographic, injury-related, and clinical information of patients admitted at two trauma centers in Cali, Colombia.

Study design: Descriptive observational study, using trauma registries implemented in two trauma centers in Cali, Colombia, between January 1st and August 31st 2012.

Results: From 11,889 trauma admissions, there were 1395 $(11.6 \%)$ injuries were related to MVCs. Injury severity scores $<9$ were found in $86 \%(n=1200)$ of the patients; from these, $48.4 \%$ $(n=675)$ were injured in motorcyclists, $26.8 \%(n=374)$ were bicyclists, and $10.3 \%(n=144)$ were vehicle occupants. Blunt trauma was the most frequent type of injury $(93.5 \%, n=1304)$. 
After stratification by age, it was observed that motorcyclists were the most often associated to injuries related to MVCs, with regards of patients between 5 and 14 years of age. There were $777(55.7 \%)$ patients injured because of MVCs that required hospitalization and there were $114(14.7 \%)$ patients admitted to intensive care units. Overall mortality was $2.7 \%$ (53 patients).

Conclusion: In Cali, Colombia, injuries related to motor vehicle crashes are mostly young men, economically active, driving motorcycles and suspected to have drinking alcohol prior the events. We hope these findings will contribute to control and prevention strategies against traffic-related injuries in the local context and in contexts similar to those of Cali.

Keywords: Trauma, Vehicular, Motorcycle, Alcohol, Motor vehicle crash.

\section{INTRODUCCIÓN}

El trauma debido a lesiones relacionadas con colisiones de vehículos motorizados es definido como "cualquier trauma debido a un incidente originándose, terminando o involucrando un vehículo motorizado parcial o totalmente en una vía pública." Cada día mueren más de 3000 personas por lesiones de tránsito en todo el mundo. Al año, 1.2 millones mueren y otros 50 millones resultan con heridas. ${ }^{1}$ Esta problemática afecta más a los países de ingresos bajos y medianos, que son los más afectados según el informe de prevención de los eventos de tránsito de la OMS, concentrándose el $85 \%$ de la mortalidad por trauma debido a eventos de tránsito y $90 \%$ de los años de vida ajustados en función de discapacidad (AVAD). En 1990, los AVAD por trauma debido a lesion relacionada con colision de vehículos motorizados ocupaban la novena posición y para el 2020 se espera que ocupen la tercera. ${ }^{1}$

En Colombia las muertes por trauma debido a lesiones relacionadas con colisiones de vehículos motorizados son una causa importante de muerte y morbilidad, que cobran importancia debido a que son prevenibles. ${ }^{2}$ Según los datos encontrados desde 2002-2011 diariamente 16 personas mueren y otras 111 terminan con algún tipo de trauma debida a estas lesiones. ${ }^{3}$ En el 2012 el Ministerio de Salud publicó que se presentaron 6152 muertes asociadas a trauma por lesion relacionada con colision de vehículos motorizados, que comparado con los dos años inmediatamente anteriores (5792 muertes en 2011 y 5704 muertes en 2010) representa un aumento del 6,2\% de la mortalidad debida a lesión relacionada con colision de vehículos motorizados, lo que según el Ministerio de Salud es la cifra más alta que se ha presentado desde hace más de 10 años (3-5).

En la ciudad de Cali entre el 2007 al 2010 el número total lesiones relacionadas con colision de vehículos motorizados se ha mantenido alrededor de 22000 por año, y entre 2011 y 2012 el número ascendió a 24540 por año, aumentando con esto la morbididad y mortalidad debida a lesión relacionada con colision de vehículos motorizados, siendo los motociclistas los más afectados. ${ }^{6,7}$ Teniendo en cuenta que esta situación tiene un impacto en la salud publica tanto nacional como regional, decidimos con este trabajo realizar una descripción de las personas que sufren traumatismos debido a lesión relacionada con colision de vehículos motorizados en la Ciudad de Cali, basándonos en los datos recolectados en el registro de trauma de dos centros de alta complejidad de la ciudad, en el periodo que comprende desde enero 01 hasta agosto 31 del año 2012.

\section{METODOLOGÍA}

El Registro Internacional de Trauma es una base de datos electrónica basada en web, perteneciente a la Sociedad Panamericana de Trauma mediante el Programa Internacional de Sistemas de Trauma (ITR/SPT-ITSDP); es el componente internacional de la División de Trauma y Cuidado Crítico de la Virginia Commonwealth University $(\mathrm{VCU}) .{ }^{8}$ El software del registro de trauma es una herramienta digital desarrollada para funcionar en red a través de internet, consta de un total de 244 variables distribuidas en 11 ventanas que agrupan los datos en módulos operativos: Información demográfica; motivo de consulta; antecedentes y lugar del evento; mecanismos de lesión; información prehospitalaria; examen físico; imágenes; laboratorio; diagnóstico y manejo en urgencias; hospitalización; referencia y contra-referencia. De esta forma permite registrar el seguimiento diario del paciente hospitalizado, especificar si fue o no sometido a cirugía u otros procedimientos, calcular automáticamente ciertas variables como edad de acuerdo a la fecha de nacimiento, escalas e índices como la Escala de Coma de Glasgow, el Índice de Trauma Revisado (RTS), el Índice de Severidad de las Lesiones (ISS) y el estatus al egreso de cada paciente. Permite generar reportes estandarizados y otros específicos según la necesidad de información. Todos los diagnósticos clínicos y los mecanismos de lesión están codificados según la Clasificación Internacional de Enfermedades (CIE-10 ó ICD-10 por sus siglas en inglés). ${ }^{8}$

Se realizó la selección de los sujetos identificados con trauma debido a lesión relacionada con colision de vehículos motorizados. El estudio fue aprobado por el Comité de Ética de las dos instituciones. La información depositada en el registro de trauma es capturada en equipos portátiles dotados de conexión a red inalámbrica. La captura de la información es realizada por personal técnico en atención pre-hospitalaria debidamente entrenados, que en tiempo real desde urgencias, y una vez el paciente es trasladado las diferentes salas del hospital, los datos son adquiridos de la historia clínica; documento físico en el hospital público y en documento electrónico (SAP NetWeaver Business Client 1.0 $\mathrm{SAP}^{\circledR}$ ) en el hospital privado. Se incluyen todos los pacientes que ingresan a ambos hospitales por causa de atención al 
trauma. Los datos para el análisis estadístico se exporta a una base de datos en un archivo binario, con previa autorización del centro coordinador central en la Universidad de Virginia. Este archivo es transferido a la plataforma de análisis en el software estadístico STATA ${ }^{\mathrm{TM}} 10$ (StataCorp, Texas-USA). Se realiza un análisis exploratorio descriptivo; las variables categóricas son presentadas en proporciones y las comparaciones entre ellas se realizarán con chi cuadrado o prueba exacta de Fisher según el caso. Las variables continuas se expresan como media y desviación estándar o mediana y rangos intercuartílicos; estas son analizados con Student's T test ó Mann-Whitney test, según su distribución.

\section{RESULTADOS}

En las dos instituciones se realizó la atención de 11889 pacientes con trauma en un periodo de 8 meses, de los cuales $1395(11,7 \%)$ corresponden a pacientes con lesiones relacionadas a colision de vehículos motorizados, atendidos en mayor proporción en el hospital público (PUB) $n=777$ $(55,7 \%)$ que en el privado [(PRI) $n=618(44,3 \%), p<0,0001)]$.

La mediana de la edad de los sujetos fue de 28 años (rango inter cuartil [RIC] 19-41) y la mayoría eran hombres [1012 (72,6\%)], con una proporción mayor en el hospital público comparado con el privado [PUB $=592(76,3 \%) v s$ $420(68,0 \%), \mathrm{p}<0,0006]$.

La severidad del trauma fue calculada mediante el ISS, donde de acuerdo a las tres categorías utilizadas (ISS $<9$, 9-15 y >15) el comportamiento fue similar entre los dos hospitales, siendo más frecuente encontrar sujetos con ISS $\leq 9(1200(86,0 \%))$.

El mecanismo de trauma contuso fue observado en 1304 $(93,5 \%)$ sujetos, con una proporción mayor atendida en el hospital privado $[\mathrm{PUB}=744(94,6 \%) v s \mathrm{PRI}=560$ (99,06\%), $\mathrm{p}=0,0001]$; las heridas penetrantes fueron encontradas en $33(2,4 \%)$ pacientes, con una proporción mayor atendida en el hospital público [PUB = $27(3,5 \%)$ vs $\mathrm{PRI}=6(1,0 \%)$, $\mathrm{p}=0,0022]$ (Tabla 1).

El consumo de sustancias psicoactivas fue encontrado en 192 (13,8\%) pacientes en el momento de la atención. En el hospital público se encontraron cuatro pacientes con sospecha de consumo de bazuco y en 1 paciente sospecha de consumo de sustancias inhaladas. Se sospechó consumo de alcohol en el momento de la atención en 187 (16,7\%) pacientes, esto fue más frecuentemente observado en el hospital público $(\mathrm{PUB}=164 / 671(24,4)$ vs $\mathrm{PRI}=23 / 447$ $(5,2), \mathrm{p}<0,0001]$.

Del total atendido por lesión relacionada con colision de vehículos motorizados, 675 (48,4\%) personas usaban motocicletas como medio de transporte, $374(26,8 \%)$ personas reportaban usar bicicleta como medio de transporte en el momento y 144 (10,3\%) personas vehículos livianos (Tabla 2).

Realizando la división de estos pacientes en distintos grupos de edad (Tabla 3), encontramos que en el grupo de 0 a 4 años se observó más frecuentemente la motocicleta en 17 (44,7\%) como principal medio de transporte relacionado con lesiones relacionadas a colision vehícular motorizada, seguido de la bicicleta en $11(10,5 \%)$ pacientes y el vehículo liviano en $4(10,5 \%)$. En el grupo de pacientes entre 5 a 14 años se encontró más frecuentemente involucrada la bicicleta en 91 $(53,2 \%)$ pacientes, seguido de la motocicleta en $34(19,9 \%)$ pacientes y el vehículo liviano en $15(8,8 \%)$ pacientes. En los grupos de pacientes que abarcan las edades entre los 15 a 74 años, se encontró que se presentan 1147 (82\%) de las lesiones relacionadas con colision de vehículos motorizados, con un patrón similar en el tipo de vehículo con el cual tenían algún tipo de relación directa o indirecta, donde fue la motocicleta el medio de transporte relacionado más común en 614 (53\%) pacientes, seguido por la bicicleta en $261(22 \%)$ pacientes y el vehículo liviano en 123 (10\%) pacientes. En los pacientes mayores a 75 años el trauma debido a lesión relacionada con colision de vehículos motorizados fue más frecuentemente

Tabla 1: Características generales de los pacientes, severidad, mecanismo de trauma y consumo de alcohol de acuerdo al sitio de atención

\begin{tabular}{|c|c|c|c|c|}
\hline & $\begin{array}{l}\text { Total } \\
(n=1395)\end{array}$ & $\begin{array}{l}P U B \\
(n=777)(55,7)\end{array}$ & $\begin{array}{l}P R I \\
(n=618)(44,3)\end{array}$ & $p$-value \\
\hline Edad (años)* & $28(19-41)$ & $27(19-42)$ & $29(20-41)$ & 0,3287 \\
\hline Sexo (hombres) & $1012(72,6)$ & $592(76,2)$ & $420(68,0)$ & 0,0006 \\
\hline \multicolumn{5}{|l|}{ ISS } \\
\hline$\leq 9$ & $1200(86,0)$ & $670(86,2)$ & $530(85,8)$ & 0,802 \\
\hline $9 \leq 15$ & $63(4,5)$ & $37(4,8)$ & $26(4,2)$ & 0,6201 \\
\hline$>15$ & $129(9,2)$ & $69(8,9)$ & $60(9,7)$ & 0,5957 \\
\hline \multicolumn{5}{|c|}{ Mecanismo del trauma } \\
\hline Contuso & $1304(93,5)$ & $744(94,6)$ & $560(99,06)$ & 0,0001 \\
\hline Penetrante & $33(2,4)$ & $27(3,5)$ & $6(1,0)$ & 0,0022 \\
\hline
\end{tabular}

*Mediana (RIC) 
Epidemiología de Lesiones Relacionadas con Colisiones de Vehículos Motorizados en dos Centros de Referencia

Tabla 2: Tipo de vehículo relacionado con la atención del paciente

\begin{tabular}{lll}
\hline Tipo de Vehículo & $n$ & $\%$ \\
\hline Motocicleta & 675 & 48,4 \\
Bicicleta & 374 & 26,8 \\
Vehículo liviano & 144 & 10,3 \\
Camión/Camioneta/Bus & 86 & 6,2 \\
Peatón & 67 & 4,8 \\
Otro & 49 & 3,5 \\
\hline
\end{tabular}

relacionado con el uso de bicicleta en 11 pacientes (30,6\%), seguido de la motocicleta en 10 pacientes $(27,8 \%)$ en motocicleta y 16 pacientes $(16,7 \%)$ eran peatones.

Del total de pacientes atendidos en urgencias, fueron hospitalizados $777(55,7 \%)$ y $114(8,2 \%)$ requirieron ser llevados a la unidad de cuidado intensivo (Tabla 4). La mediana de estancia global en hospitalización fue de 3 días $(\mathrm{RIC}=1-7)$, y la mediana de estancia global en la unidad de cuidados intensivos fue de 6 días (RIC=3-12) (see Tabla 4).

La mortalidad global registrada fue de $53(2,7 \%)$ pacientes, de estos $6(0,4 \%)$ pacientes murieron en el servicio de urgencias y $47(6 \%)$ pacientes durante su estancia hospitalaria (see Tabla 4).

\section{DISCUSIÓN}

Según reportes de la OMS, el trauma debido a lesión relacionada con colision de vehículos motorizados en el año 2010 se desplazó hacia los primeros lugares en la escala de los eventos relacionados a carga de la enfermedad a nivel mundial, ocupando el tercer lugar después de la enfermedad coronaria y la enfermedad unipolar depresiva grave, aportando 1,2 millones de muertes anuales y representando el $11 \%$ de la carga total de enfermedad a nivel mundial; representando solo la punta del iceberg. ${ }^{1,3,4,9}$ En el año 2010, se ha descrito que el trauma debido a lesión de tránsito genero 74872 AVAD, en comparación con los generados por la enfermedad cardiaca hipertensiva de 15324 AVAD. ${ }^{9}$

En el marco del reconocimiento de estas lesiones como un problema de salud pública, la OMS ha priorizado dentro de sus objetivos la caracterización epidemiológica de la población que sufre de trauma asociado a lesión relacionada con colision de vehículos motorizados para mejorar la comprensión de este fenómeno y construir herramientas para el desarrollo de estrategias de intervención. ${ }^{1}$ Esta posición de la OMS se relaciona con las proyecciones para el año 2020, que refieren de un aumento de hasta el $80 \%$ en la mortalidad por trauma asociado a lesión relacionada con colision de vehículos motorizados. ${ }^{10}$

En este sentido algunos estudios internacionales han identificado algunas características de este tipo de trauma en las poblaciones, entre las cuales: una relación inversamente proporcional entre el automotor involucrado y el nivel de ingreso económico del país en el cual se presenta; en Colombia considerado un país de ingresos medios-bajos las motocicletas son los vehículos más frecuentemente involucrados en estos tipos de traumatismos. ${ }^{2,11-13}$ Esta variación de las características entre las regiones en donde se presentan las lesiones, puede estar relacionada al igual que otras lesiones por trauma, a la variación del comportamiento de acuerdo a las características, al tiempo y a la región en donde ocurren. ${ }^{13-24}$

Los estudios realizados en Cali han reportado tasas de mortalidad por lesión relacionada con colision de vehículos motorizados comparativamente elevadas, ya que en 1987 la tasa fue de 16 por 100,000 personas, para 1992 de 29 por 100,000 personas, para el 2008 de 18 por 100,000 personas y para el 2012 de 13 por 100,000 personas, ${ }^{25}$ con esto superando las de otras ciudades importantes de Colombia como Bogotá (7 por 100,000), Medellín (11 por 100,000) y Barranquilla (12 por 100,000) $(5,25)$, lo que se podría explicar que en Cali para el 2012 esta fuera la novena causa de mortalidad global reportada $(5,25)$.

Entre los años 2007 al 2010 según el reporte anual local el número de eventos de tránsito y la morbi-mortalidad relacionadas permanecieron estables, pero entre los años 2011 a 2012 se encuentra un ascenso en el número total de estos eventos de tránsito y la morbi-mortalidad relacionada, ${ }^{5,6}$ razón por la cual se deberían aumentar y reforzar las medidas preventivas ya establecidas, ${ }^{26}$ pues desde hace varios años está demostrado que los eventos de tránsito son fenómenos

Tabla 3: Tipo de vehículo relacionado trauma debido a lesión de tránsito por grupos de edad

\begin{tabular}{|c|c|c|c|c|c|c|c|c|c|c|c|c|c|}
\hline \multirow[t]{2}{*}{ Edad } & \multicolumn{2}{|c|}{ Bicicleta } & \multicolumn{2}{|c|}{ Camión/Bus } & \multicolumn{2}{|c|}{ Motocicleta } & \multicolumn{2}{|c|}{ Vehículo Liviano } & \multicolumn{2}{|c|}{ Otro } & \multicolumn{2}{|c|}{ Peatón } & \multirow[t]{2}{*}{ Total } \\
\hline & $n$ & $\%$ & $n$ & $\%$ & $n$ & $\%$ & $n$ & $\%$ & $n$ & $\%$ & $n$ & $\%$ & \\
\hline $0-4$ & 11 & 28,9 & 1 & 2,6 & 17 & 44,7 & 4 & 10,5 & 3 & 7,9 & 2 & 5,3 & 38 \\
\hline 5 a 14 & 91 & 53,2 & 13 & 7,6 & 34 & 19,9 & 15 & 8,8 & 5 & 2,9 & 13 & 7,6 & 171 \\
\hline 15 a 29 & 117 & 21,6 & 26 & 4,8 & 319 & 58,9 & 50 & 9,2 & 17 & 3,1 & 13 & 2,4 & 542 \\
\hline 30 a 44 & 73 & 21,2 & 20 & 5,8 & 191 & 55,4 & 38 & 11,0 & 5 & 1,4 & 18 & 5,2 & 345 \\
\hline 45 a 59 & 52 & 28,0 & 16 & 8,6 & 83 & 44,6 & 24 & 12,9 & 4 & 2,2 & 7 & 3,8 & 186 \\
\hline 6074 & 19 & 25,7 & 5 & 6,8 & 21 & 28,4 & 11 & 14,9 & 11 & 14,9 & 7 & 9,5 & 74 \\
\hline$\geq 75$ & 11 & 30,6 & 5 & 13,9 & 10 & 27,8 & 2 & 5,6 & 2 & 5,6 & 6 & 16,7 & 36 \\
\hline
\end{tabular}




\begin{tabular}{lll}
\hline \multicolumn{3}{c}{ Tabla 4: Hospitalización, cuidado crítico y mortalidad } \\
\hline & $n$ & $\%$ \\
\hline Hospitalizados & 777 & 55,7 \\
Días $^{*}$ & $3(1-7)$ & \\
ICU & 114 & 8,2 \\
Días $^{*}$ & $6(3-12)$ & \\
Muerte en urgencias & $6 / 1395$ & 0,4 \\
Muerte en hospitalización & $47 / 777$ & 6,0 \\
General & $53 / 1935$ & 2,7 \\
\hline
\end{tabular}

*Mediana (RIC)

totalmente prevenibles, y que por lo tanto el termino de accidente de transito no sería el apropiado. ${ }^{1}$

Sin embargo, la información sobre las características de este fenómeno y las descripciones epidemiológicas locales son escasas, en algunas publicaciones se pueden identificar algunas características de manera indirecta, por ejemplo, en un estudio realizado en dos centros nivel IV de atención, el porcentaje de pacientes atendidos por lesiones de tránsito se encuentra alrededor del $12 \% .{ }^{6}$ Dado el escenario local y la problemática actual, se realizó la conducción de este estudio para caracterizar los pacientes con lesiones relacionadas con colision de vehículos motorizados, identificando principalmente el evento en hombres jóvenes, en edades económicamente productivas e involucrando más frecuentemente el uso de motocicletas; estas características demográficas de la población son similares a otras descripciones. ${ }^{1,14}$

El uso de las motocicletas y del comportamiento entre los grupos de edades pueden estar relacionados con la información identificada por entidades oficiales y no oficiales, en la cual se señala que en el año 2012, las motocicletas fueron el medio de transporte particular más frecuentemente empleado en la ciudad (32\%); con un total de 150978 motocicletas registradas y en circulación activa y en su mayoría manejadas por hombres (83\%) en edades económicamente productivas $(93 \%)$ y usada como medio de transporte para desplazarse a sus actividades

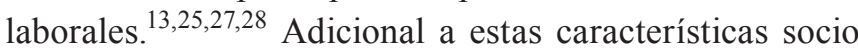
demográficas mencionadas, un estudio nacional identifico que el $65 \%$ de los usuarios de las motocicletas son jefes de hogar, ${ }^{13}$ entonces ya involucrando el papel económico familiar.

Llama la atención que en el grupo de edad entre 0 a 4 años fuera la motocicleta la mas frecuentemente asociada con las lesiones relacionadas a colisiones de vehículos motorizados, pues en Colombia el transporte de niños se encuentra regulado por leyes de tránsito, en las que se prohíbe el transporte de todo menor de 10 años en motocicleta. ${ }^{34}$ Este fenómeno ya ha sido descrito en otros estudio en los cuales relacionan este tipo de lesiones vehiculares con personas que bajo el efecto de sustancias como el alcohol transportan a menores de edad,,$^{13,29-33}$ pero el alcance de este estudio no nos permite llegar a esta misma u otra conclusión.
Por otro lado, en el grupo de edad entre los 5 a 14 años ya se encontró la bicicleta como el vehículo mas frecuentemente asociado con lesiones relacionadas con colisión de vehículos motorizados. Este fenómeno también se ha descrito en otros estudios y se ha relacionado el uso de bicicleta con comportamientos riesgosos en cruces de calles, falta de cuidado por parte de cuidadores de menores de edad, y uso de sustancias como el alcohol en los casos de lesiones relacionadas con colisión de vehículos motorizados. ${ }^{29,35}$

En el grupo de edad de las personas mayores de 75 años, se encontró la bicicleta como el vehículo mas frecuentemente asociado con lesiones relacionadas con colisión de vehículos motorizados. Teniendo e cuenta a este como un grupo de alto riesgo para lesiones de tránsito, debido el deterioro en las capacidades de reacción y movilidad debidas a la edad, es importante realizar mayores medidas preventivas ajustadas a las características de esta población. ${ }^{36}$

En cuanto a los mecanismo de trauma, el mecanismo más frecuentemente observado fue el trauma cerrado con una mortalidad general de $2,7 \%$, similar a lo ya descrito por otros estudios. ${ }^{3,21-24,37,38}$ Las áreas anatómicas más comúnmente comprometidas fueron las craneoencefálicas y las clasificadas como traumatismo múltiple, esto también siguiendo el mismo comportamiento descrito en otras publicaciones. ${ }^{3,4,12,39}$ La severidad del compromiso que más se encontró fue la identificada como trauma menor y el 10\% como traumas severos. ${ }^{40}$

A pesar de que ya se ha demostrado que el consumo de alcohol aumenta el riesgo de tener una lesión relacionada con colisión de vehículos motorizados, en Colombia estudios recientes muestran que el $12 \%$ de los que sufren este tipo de traumatismo se encuentran bajo efectos del alcohol, además de que los días donde mas se presentan estas lesiones son el sábado y el domingo, en horas de la madrugada, y de estos el $50 \%$ utilizaba la motocicleta como medio de transporte. . $^{3,4,15-20,24,25,41}$

Este patrón Nacional tiene un comportamiento similar en Cali, salvo el consumo de alcohol encontrado en pacientes con traumatismos debido a lesiones relacionadas con colisión de vehículos motorizados es mayor, de alrededor del $40 \%$, y en nuestra experiencia aunque no se realizaron medidas confirmatorias apropiadas, se sospechó consumo de alcohol en $13.8 \%$ de los pacientes atendidos, ${ }^{18,25}$ lo que es un número inferior, pero que no es más que una sub-representación del total de personas que sufren este tipo de lesiones, pues en la ciudad de Cali las personas que sufren cualquier tipo de traumatismo asociado a lesión relacionada con colisión de vehículos motorizados son dirigidas inicialmente a otros servicios de urgencias de hospitales de nivel inferior, para definir según la severidad del trauma el sitio de atención. 
En la literatura se encontraron reportes de intervenciones internacionales exitosas, que han centrado sus políticas de intervención social en políticas de crecimiento de ciudades inteligentes, implementando normas de calidad en la producción de vehículos, implementando y haciendo cumplir normas de seguridad en carretera, reduciendo el tiempo de atención a personas lesionadas y mejorando la calidad en atención medica en estos eventos. ${ }^{1,24,42,43}$ En la ciudad de Cali se han implementado una serie de medidas preventivas como la educación a los conductores, el control del tránsito, las campañas de concientización ciudadana, la capacitación de agentes de tránsito y la implementación del plan de seguridad vial (uso obligatorio de casco, chaleco, cinturón), los cuales han mostrado una reducción parcial de la mortalidad de 406 casos en el año 2000 a 272 casos en el año 2012, con una tendencia global que sigue el patrón de otros países desarrollados que han sido exitosos en el plan de reducción de lesiones relacionadas con colisión de vehículos motorizados. ${ }^{2,10,25,44-48}$ Esperamos que los resultados de este estudio ayuden a contribuir a los esfuerzos locales de control y prevención de lesiones relacionadas con colisión de vehículos motorizados.

\section{REFERENCIAS}

1. Salud $\mathrm{OM}$ de la. Informe mundial sobre prevención de los traumatismos causados por el tránsito 2004.

2. Traffic-related injury prevention interventions for low-income countries. Inj Control Saf Promot [Internet]. 2003 Mar 1;10(1-2): 109-118. Available at: http://pubget.com/paper/12772494.

3. Instituto Nacional de Medicina Legal y Ciencias forenses. Forensis, Datos para la vida, Bogota. Division de Referencia de informacion Pericial 2011.

4. Instituto Nacional de Medicina Legal y Ciencias forenses. Forensis, Datos para la vida, Bogota. Division de Referencia de informacion Pericial 2010.

5. Lucía S, Lozada M. Instituto Nacional de Medicina Legal y Ciencias forenses. Forensis 2012, Datos para la vida, Bogota. Division de Referencia de informacion Pericial. Forensis 2012. 2012;349-402.

6. Secretaría de Salud Pública Municipal de Cali. Boletín Estadístico. Cali: Oficina de Estadística 2003.

7. Morales GE. Cali En Cifras 2012, Rerporte Anual. Bol. Secr. Salud Santiago Cali 2012.

8. Sociedad Panamericana de Trauma, Registro Panamericano de Trauma.

9. Murray CJL, Vos T, Lozano R, Naghavi M, Flaxman AD, Michaud C, et al. Disability-adjusted life years (DALYs) for 291 diseases and injuries in 21 regions, 1990-2010: a systematic analysis for the Global Burden of Disease Study 2010. Lancet [Internet]. 2012 Dec 15 [cited 2013 Feb 28];380(9859):21972203. Available at: http://www.ncbi.nlm.nih.gov/pubmed/ 23245608.

10. Espitia-hardeman V, Vélez L, Muñoz E, Gutiérrez-martínez MI. Efectos de las intervenciones diseñadas para prevenir las muertes de motociclistas en Cali , Colombia (1993-2001) 2008; 50:69-77.
11. Carlos J, Sabaini G, Martner R. America Latina: Panorama Global de su Sistema Tributario Y Principales Temas de Política. 2005;1-25.

12. Hurt HH. Motorcycle accident cause factors and identification of countermeasures: Technical Report 1981;1(1).

13. Japonesas C de E de M. Quinto Estudio Sociodemográfico de los usuarios de motos en Colombia / Perfil de usuario de 2009.

14. Colombia $\mathrm{C}$ de $\mathrm{E}$ de $\mathrm{M}$ de. Las motos: Aliadas del desarrollo de Colombia 2006;1-13.

15. En C de PS, Social AS de PSM de D. Consumo de alcohol observatorio coordinación de Políticas Sociales. 2009;1-9.

16. GS S, CC B, TR M. Fatal nontraffic injuries involving alcohol: A metaanalysis. Ann Emerg Med. 1999 Jun;33(6):659-668. [Internet]. 1999 Jun 1;33(6):659-668. Available at: http://pubget. com/paper/10339681.

17. Odero W, Garner P, Zwi A. Road traffic injuries in developing countries: a comprehensive review of epidemiological studies. Trop Med Int Heal 1997 May;2(5):445-460 [Internet]. 1997 May 1;2(5):445-460. Available at: at://pubget.com/paper/9217700.

18. Hingson R, Heeren T, Winter M, Wechsler H. Magnitude of alcohol-related mortality and morbidity among US college students ages 18-24: changes from 1998 to 2001. Annu Rev Public Heal. [Internet]. 2005 Jan 1;26:259-279. Available at: http://pubget.com/paper/15760289.

19. JA van O, HF G. The geographic relationship between alcohol use, bars, liquor shops and traffic injuries in Rotterdam. J Stud Alcohol [Internet]. 1993 Nov 1;54(6):739-744. Available at: http://pubget.com/paper/8271811.

20. Chisholm D, Rehm J, M VO, Monteiro M. Reducing the global burden of hazardous alcohol use: a comparative cost-effectiveness analysis. J Stud Alcohol [Internet]. 2004 Nov 1;65(6):782-793. Available at: http://pubget.com/paper/ 15700517.

21. Rehm J, Sulkowska U, Mańczuk M, Boffetta P, Powles J, Popova $\mathrm{S}$, et al. Alcohol accounts for a high proportion of premature mortality in central and eastern Europe. Int J Epidemiol [Internet]. 2007 Apr 1;36(2):458-467. Available at: http://pubget. com/paper/17251244.

22. Rehm J, Patra J, Popova S. Alcohol-attributable mortality and potential years of life lost in Canada 2001: implications for prevention and policy. Addiction [Internet]. 2006 Mar 1;101(3):373-384. Available at: http://pubget.com/paper/ 16499510.

23. Rehm J, Room R, Monteiro M, Gmel G, Graham K, Rehn N, et al. Alcohol as a risk factor for global burden of disease. Eur Addict Res [Internet]. 2003 Oct 1;9(4):157-164. Available at: http://pubget.com/paper/12970584.

24. Rehm J, Gmel G, CT S, Trevisan M. Alcohol-related morbidity and mortality. Alcohol Res Heal. [Internet]. 2003 Jan 1;2(1):39-51. Available at: http://pubget.com/paper/15301399.

25. Secretaria de Gobierno C y S. Violencia, convivencia y dinamica social en cali: lectura desde el observatorio social 2011.

26. Cisalva. Observatorio de muertes por lesiones de causa externa área metropolitana de cali.

27. Nacional S de T. Motos Matriculadas en Colombia por Organismo de Tránsito 2010.

28. Dias J. Horarios de accidentes: Las horas mas peligrosas. Crash test/Segur Vial 2008;1:6-10.

29. Arias Cohl S. Factores de riesgo asociados a accidentes de tránsito en menores de 19 años. Pediatr. (Asunción), 2005; 32(1):16-22. ISSN 1683-9803. 2005. 
30. Ahlm K, Eriksson A. Driver's alcohol and passenger's death in motor vehicle crashes. Traffic Inj Prev [Internet]. $2006 \mathrm{Sep}$ 1;7(3):219-223. Available at: http://pubget.com/paper/16990235.

31. Child Passenger Deaths Involving Drinking Drivers-United States, 1997-2002. 2004 Feb 25;291(8):934-345. Available at: http://pubget.com/paper/22596719.

32. LH M, Kotch J, JH L. Children in alcohol-related motor vehicle crashes. Pediatrics [Internet]. 1986 Jun 1;77(6):870-872. Available at: http://pubget.com/paper/3714380.

33. HM S, DR M, RA W. Epidemiology of road accidents involving young adults: alcohol, drugs and other factors. Drug Alcohol Depend [Internet] 1982 Sep 1;10(1):35-63. Available at: http:/ pubget.com/paper/7173041.

34. Nacional S de T. Ley 1383 del 2010. Minist Transp 2010;1-30.

35. Trends in motorcycle fatalities associated with alcohol-impaired driving-United States, 1983-2003. MMWR Morb Mortal Wkly Rep [Internet] 2004 Dec 3;53(47):1103-1106. Available at: http:// pubget.com/paper/15573026.

36. Nelci Astrid Becerra Martínez, Andrés Felipe Cadena Bastidas Lhat. Prevención de accidentes en adultos mayores 2007.

37. AristizábalD, GonzálezG, SuárezJF, Roldán P. Factores asociados al trauma fatal en motociclistas en Medellín 2005-2008. 2012.

38. Piat. Lesiones más frecuentes asociadas a accidentes de tránsito; severidad, letalidad y factores asociados a esta severidad y letalidad. Salud Sin Límites Perú 2009;26.

39. Association EMM. European motorcycle manufacturers association. MAIDS In-depth investigations of accidents involving powered two wheelers. Final Report 1.3. Bruselas. 2008.

40. Holbrook TL, Hoyt DB. The impact of major trauma: quality-oflife outcomes are worse in women than in men, independent of mechanism and injury severity. J Trauma 2004 Feb;56(2):284-290.
41. Cherpitel CJ. Alcohol and injuries: a review of international emergency room studies since 1995. Drug Alcohol Rev. [Internet] 2007 Mar [cited 2013 Apr 2];26(2):201-214. Available at: http://www.ncbi.nlm.nih.gov/pubmed/17364856.

42. AL D, SP B, Li G. Intentional and unintentional injuries in women. An overview. Ann Epidemiol [Internet] 1994 Mar 1; 4(2):133-139. Available at: http://pubget.com/paper/8205280.

43. Peniche L RA. Psicología de los accidentes. Primera edición. México: Editorial CECSA 1985.

44. Crompton JG, Bone C, Oyetunji T, Pollack KM, Bolorunduro $\mathrm{O}$, Villegas C, et al. Motorcycle helmets associated with lower risk of cervical spine injury: debunking the myth. J Am Coll Surg [Internet] American College of Surgeons 2011 Mar [cited 2013 Apr 14];212(3):295-300. Available at: http://www.ncbi. nlm.nih.gov/pubmed/21227720.

45. BC L, Ivers R, Norton R, Boufous S, Blows S, SK L. Helmets for preventing injury in motorcycle riders. Cochrane Database Syst Rev [Internet] 2008 Jan 1;(1):CD004333-CD004333. Available at: http://pubget.com/paper/18254047.

46. Liu B, Ivers R, Norton R, Blows S, SK L. Helmets for preventing injury in motorcycle riders. Cochrane Database Syst Rev [Internet] 2004 Jan 1;(2):CD004333-CD004333. Available at: http://pubget.com/paper/15106247.

47. FL M, LL G. Statistical analysis of motorcyclists' perceived accident risk. Accid Anal Prev [Internet] 1995 Feb 1;27(1):21-31. Available at: http://pubget.com/paper/7718075.

48. Sirathranont J, Kasantikul V. Mortality and injury from motorcycle collisions in Phetchaburi Province. J Med Assoc Thai [Internet] 2003 Feb 1;86(2):97-102. Available at: http:// pubget.com/paper/12678145. 